\title{
Intestinal Parasitic Infections among Egyptian Patients with Chronic Liver Diseases at Zagazig University Hospital
}

\author{
Asmaa Mohammed Farouk Al-Ghandour ${ }^{1}$, Abeer Hussein Abdelkader ${ }^{2}$, \\ Hytham Kamal Ahmed ${ }^{3}$, Ehab M Darwiesh², Howayda Said \\ Fouad Moawad ${ }^{1}$ \\ ${ }^{1}$ Department of Medical Parasitology, Faculty of Medicine, Zagazig University,Egypt. \\ ${ }^{2}$ Department Tropical Medicine, Faculty of Medicine, Zagazig University, Egypt. \\ ${ }^{3}$ Department Clinical Pathology, Faculty of Medicine, Zagazig University, Egypt.
}

Corresponding Author Al-Ghandour, Asmaa Mohammed Farouk

Mobile:

01140746382

E mail:

asmaamfarouk@gmail. com

Key words:

Diarrhea, (CLD), Cryptosporidium spp., Giardia, ELISA, immune status
Background and study aim: Regarding the increased number of chronic liver diseases (CLD) patients suffering from many manifestations e.g. diarrhea, this withdrew our attention to try to find a relation between CLD and parasitic infections in those patients.

Patients and Methods: A case-control study was performed on 190 participants suffering from gastrointestinal complaints especially diarrhea attending Gastroenterology and Hepatology Outpatient Clinics at Zagazig University Hospital, divided into 2 groups GI: 95 CLD patients. GII: 95 non-CLD Control group patients. Cases underwent clinical, abdominal ultrasonographic, and laboratory liver function assessments. Also, collected patients' stool samples were microscopically examined using iodine, Trichrome and Modified ZiehlNeelsen stains. To confirm accurate diagnosis to parasitic infections causing diarrhea, RIDA-ELISA for Cryptosporidium spp. and Giardia lamblia copro-antigens detections was performed.
Results: Parasitic infections among both studied groups were $(47 \%)$, comprising $(58 \%)$ in CLD and (36\%) in non-CLD as follow: Cryptosporidium (28\%, 14\%), Giardia (15\%, 12\%), mixed (5\%, 3\%), E.histolytica spp. (5\%, 4\%), Blastocystis hominis $(3 \%, 1 \%)$ and $H$. nana $(1 \%, 2 \%)$ respectively. The sensitivity, specificity, PPV, NPP and accuracy of ELISA regarding Giardia and Cryptosporidium infections were $(100 \%$ and $89.6 \%, 97.5 \%$ and $100 \%, 87.9 \%$ and $100 \%, 100 \%$ and $96.6 \%, 97.9 \%$ and $98.4 \%$ ) respectively. In GI, most giardial cases had normal ALT and AST levels $(74 \%, 63 \%)$, but elevated in cryptosporidial infection $(59 \%, 66 \%)$ respectively, with statistically significant difference.

Conclusion: Presence of intestinal parasitic infections; mainly Cryptosporidia and Giardia protozoa among CLD patients was striking when compared to diarrheic non-CLD control group and this may be attributed to impaired immune status.

\section{INTRODUCTION}

Chronic liver diseases (CLD) encompass a major health problem worldwide. It is one of the major causes of death that has a year-onyear rising incidence [1]. CLD refer to a long-term pathological process of continuous destruction of liver parenchyma and its gradual substitution with fibrous tissue [2]. They comprise progressive deterioration of liver functions for more than six months, which includes synthesis of clotting factors, other proteins, detoxification of harmful products of metabolism and excretion of bile. During which, continuous process of inflammation, destruction, and regeneration of liver parenchyma occur [3]. CLD can progress through stages of fibrosis to the end-stage disease, cirrhosis. Cirrhosis is an irreversible process of fibrosis, damage and regeneration of hepatocytes, altered hepatic architecture, and decreased hepatic function. Patients with CLD may 
present with cholestasis or anicteric cirrhotic liver disease [4]. Major causes of worldwide CLD are alcohol-abuse, obesity/metabolic disease, autoimmune hepatitis, and viral hepatitis (HBV and HCV); moreover, the majority of cases dying from CLD are under the age of 70 . Although there are now highly effective treatments for $\mathrm{HCV}$, by contrast there is no effective treatment for alcoholic or non-alcoholic fatty liver diseases (ALD and NAFLD), which represent the major causes of CLD in the developed world. In Egypt, CLD; mainly infective viral hepatitis HCV either denovo or on top of post-schistosomiasis liver fibrosis; that may be complicated to portal hypertension and cirrhosis, represents a common health concern according to reports from the 2008 and 2015 Egypt Demographic Health Surveys [5,6].

It is well-known that the liver has a chief role in immunity, so patients with CLD usually suffer from defects in the immune system and are highly susceptible for various microbial infections; viral, bacterial, and parasitic infections. Diminished immune response in CLD may increase the vulnerability to catch enteric parasitic infections. Both humoral and cellmediated immunity are depressed which increase with the advance of the diseases. Those patients typically have alterations in the enteric flora, reduction in serum bactericidal, opsonic activity, complements, and fibronectin levels [7]. Intestinal parasitic infections are most predominantly aggravated among patients with deficient immune status $[\mathbf{8 , 9 , 1 0}$. Patients with CLD are susceptible to a wide spectrum of parasitic infections such as opportunistic protozoa as Giardia lamblia, Entamoeba spp., Blastocystis hominis, Cryptosporidium spp., Cyclospora, Isospora belli and Microsporidia. Patients with parasitic infections suffer mostly from diarrhea, nausea, vomiting that may be aggravated to dehydration and oedema due to hypovolemia. Most of these manifestations are sharable with advanced liver disease. However, enteric protozoa as Cryptosporidium species and Giardia lamblia are the most conjoint worldwide intestinal protozoa affecting man. In developing countries cryptosporidiosis; as an opportunistic infection, hits mainly immunocompromised hosts having direct impact on chronic debilitating diseases, extending from causing only watery diarrhea to biliary tract affection and dysplastic changes of liver parenchyma $[\mathbf{1 1 , 1 2 , 1 3 ]}$. Giardiasis causes fatty, offensive, foamy diarrhea, frequently with nausea, gurgling, bloating and weight loss resulting from fat and sugar malabsorption. Chronicity may accomplish different degrees of severity of diarrhea [14]. Successful recognition and management of parasitic infections result in avoidance of the complications of diarrhea; as electrolyte disturbance, dehydration, and the expected biliary tract involvement which worsen liver status $[\mathbf{1 1 , 1 3 ]}$.

The current study focuses on the most striking parasitic infections among non-admitted patients with CLD in comparison with non-CLD; as a cause of diarrhea or other gastrointestinal tract (GIT) complaints that attract our attention to compile conventional microscopic with confirmatory RIDA-ELISA diagnosis for cryptosporidiosis and giardiasis.

\section{PATIENTS AND METHODS}

The current case-control study was carried out through the period from March 2017 to February 2018 on a sample of 190 patients with diarrhea or other GIT complaints attending the Gastroenterology and Hepatology Outpatient Clinics at Zagazig University Hospital. They completed written informed consent forms. All selected cases were subjected to full history taking including: Age, gender, residence, education attainment, socioeconomic state, medical and drug history, complaint (diarrhea, abdominal pain, anorexia, nausea, and vomiting) and clinical examination to detect pallor, jaundice, oedema, clubbing, organomegaly and ascites.

After history taking, clinical examination of all enrolled cases, they were divided into; GI: 95 patients with clinical, biochemical and sonographic evidence of (CLD). GII: Control group composed of 95 non-CLD patients of randomly-selected controls matched by age, sex and community. The inclusion criteria of recruited patients were, the age above 15 years, non-admitted out-patients suffering from diarrhea; (frequency $\geq 3$ loose stool /day) [15], either acute diarrhea (duration less than 2 weeks) [16], or chronic diarrhea (duration more than 4 weeks) [17], or other GIT complaints with exclusion of decompensated admitted cases or those who had received anti-parasite therapy in the last three months before stool specimen collection. Sample collection and dealing with 
patients followed ethical medical research guidelines and approval from Institutional Review Board (IRB) Unit, Faculty of Medicine, Zagazig University, Egypt.

\section{Parasitological study:}

In total, 3 successive fresh stool specimens from each case of 190 enrolled-patients were examined at the Parasitology laboratory of Medical Parasitology Department, Faculty of Medicine, Zagazig University. Stool samples were collected in clean sterile cups. After macroscopic examination of fecal samples including color, odor, special characters, and consistency (watery, fatty 'foamy', soft 'lacking fibers' ,mucoid, or bloody) and for the presence of living adult worms, proglottids of cestoda or undigested food, each stool sample was divided into two portions for the following investigations:

\section{(1) Microscopy:}

The first portion was examined microscopically by direct saline; wet mount preparation and/or iodine mounts to detect ova, cysts or trophozoites of parasites; and then concentration by formolethyl acetate technique [18]. Trichrome staining (the reference standard) for detecting Giardia lamblia infection was performed [19]. Modified Ziehl-Neelsen staining (the reference standard) was performed to detect intestinal coccidia; mainly Cryptosporidium spp. oocysts [20].

\section{(2) ELISA:}

The second portion was divided into 2 parts and preserved frozen at $-20{ }^{\circ} \mathrm{C}$ for further processing of the most striking parasitic infections detected by microscopy; the copro-antigens of both Giardia RIDASCREEN®Art. No.: C1101; and Cryptosporidium RIDASCREEN® Art. No.: C1201 [R-Biopharm, Germany] parasites using RIDA-ELISA enzyme immunoassay [21]. The test procedures were performed according to the instructions of the manufacturer. On adding stool specimens, antigens bound to microplates coated with Giardia and Cryptosporidium specific purified monoclonal antibodies followed by addition of conjugate. If any of these 2 protozoa were present in the specimen, a sandwich complex was formed and on addition of the substrate, there was a change in the color of the well of the plate from colorless to blue. After adding the stop reagent, the color changed from blue to yellow. The absorbance of the fecal samples was read within about 10 minutes at a wave length $450 \mathrm{~nm}$ using an ELISA micro-titer plate reader [Immunoskan-MS, Biological Diagnostic Supplies Limited, UK]. The cut-off value of the test was measured by adding 0.15 absorbance units to the measured absorption of the negative control. Samples were considered positive if their absorbance values were more than $10 \%$ above the calculated cut-off value. The samples were considered negative if the absorbance value was lower than $10 \%$ below the calculated cut-off value.

\section{Laboratory investigations:}

Measuring the liver enzymes mainly alanine transaminase (ALT) and aspartate transaminase (AST) as their elevations denote hepatocellular injuries. Normal reference range for (ALT) is 0 to $45 \mathrm{IU} / \mathrm{L}$ and (AST) is 0 to $35 \mathrm{IU} / \mathrm{L}$ [22].

\section{Abdominal Ultra-Sonography (U/S):}

Ultrasound used for assessment of the liver status, diagnosis of cirrhosis, detection of ascites and exclusion of hepatic focal lesions.

\section{Statistical analysis:}

All data were collected, tabulated and statistically analyzed using SPSS 22.0 for windows. Continuous Quantitative variables were expressed as the mean \pm SD and categorical qualitative variables were expressed as absolute frequencies "number" \& relative frequencies (percentage). Categorical data were compared using the Chi-square $\left(\chi^{2}\right)$ test. Probability $(\mathrm{P}$ value) was considered statistically significant when $<0.05[\mathbf{2 3}]$.

\section{RESULTS}

Concerning the demographic criteria of the studied groups, there were insignificant statistical differences between them regarding the gender, age and residence. Male gender represented the major percentage of both groups and also, most of them were rural inhabitants, Table (1).

The patients in CLD groups were classified into 5 classes according to the cause of liver disease. Liver cirrhosis patients represented the major group (45\%), followed by chronic hepatitis $\mathrm{C}$ (25\%), chronic hepatitis B (14\%), non-alcoholic fatty liver (11\%), and lastly autoimmune hepatitis (5\%),Table (2).

Among 190 examined cases, different presentations were detected. The most frequent gastrointestinal manifestation in the two groups was diarrhea (52\%), then bloating and flatulence 
(29\%), abdominal pain (25\%), fever (11\%), nausea and vomiting (10\%). All manifestations were significantly different between all groups except for abdominal pain, Table (3). In CLD patients, weight loss was reported in $66 \%$, splenomegaly in $57 \%$, hepatomegaly in $32 \%$, jaundice in $25 \%$, ascites in $23 \%$ and lower limb oedema in $20 \%$ of patients, Table (4).

Among 98 diarrheic patients distributed as 61 cases within CLD and 37 cases within Non-CLD group, acute diarrhea was evident in $61 \%$ of CLD patients and $39 \%$ of Non-CLD patients, while chronic diarrhea was evident in $66 \%$ of CLD patients and $34 \%$ of Non-CLD patients with non-significant difference between groups. However, high significant difference was detected $(<0.0001)$ regarding parasitic diarrhea $(71 \%)$ and non-parasitic (29\%), where the most evident parasites were Cryptosporidium spp. (53\% of acute, $21 \%$ of chronic) diarrheic cases and Giardia lamblia (26\% of acute, $10 \%$ of chronic) diarrheic cases, Table (5). The characters of stool among acute diarrheic cases represented 12\% non-mucoid soft stool (lacking fibers), only $4 \%$ mucoid stools, however watery diarrhea represented the foremost character $74 \%$ and fatty, foamy, offensive in $10 \%$ of those patients. Among chronic diarrheic patients 14\% had non-mucoid soft stool (lacking fibers), $17 \%$ mucoid stool, watery diarrhea represented $28 \%$, and fatty, foamy, offensive in $41 \%$ of those patients, with statistically high significant difference, Table (6).

The overall parasitic infection rate detected in both studied groups was (47\%). In the CLD group (58\%) of cases were infected as follow: by Cryptosporidium spp. 28\%, Giardia lamblia
15\%, mixed Cryptosporidium and Giardia 5\%, E. histolytica spp. 5\%, Blastocystis hominis 3\% and $H$. nana $1 \%$. In the non CLD group, (36\%) of cases were infected as follow: by Cryptosporidium spp. 14\%, Giardia lamblia $12 \%$, mixed Cryptosporidium and Giardia 3\%, E. histolytica spp. 4\%, Blastocystis hominis $1 \%$ and H. nana 2\%, Table (7) and Fig. (1).

Most cases of Giardia lamblia infection in CLD group had normal levels of ALT and AST (74\%, $63 \%$ ), respectively while most cases of Cryptosporidium spp. infection had elevated ALT and AST $(59 \%, 66 \%)$ respectively, with significant difference between the two infected groups, Table (8).

Concerning the RIDA-ELISA results, the negative control reading for Giardia lamblia was 0.042 . So, the cut-off value was $(0.042+0.15)$ $=0.192$. Result was considered positive if the reading was $10 \%$ more than the cut-off value $=$ $0.192+0.019=0.211$. The negative control reading for Cryptosporidium was 0.05 . So, the cut-off value was 0.2. Result was considered positive if the reading was more than $(0.2+0.02)$ $=0.22$.

RIDA-ELISA detected more cases of Giardia lamblia infection than microscopy, 4 cases were not detected by microscopy, while in Cryptosporidium spp. infection ELISA failed to detect five cases which were detected by microscopy. So, the sensitivity, Specificity, PPV, NPP and diagnostic accuracy of ELISA regarding Giardia and Cryptosporidium infections were as follow (100\% and $89.6 \%$, $97.5 \%$ and $100 \%, 87.9 \%$ and $100 \%, 100 \%$ and $96.6 \%, 97.9 \%$ and $98.4 \%$ ) respectively, Table (9).

Table (1): Demographic data of all patients in CLD and Non-CLD studied groups.

\begin{tabular}{|c|c|c|c|c|c|c|c|}
\hline \multirow{2}{*}{ Parameter } & \multirow{2}{*}{ Group } & \multirow{2}{*}{$\begin{array}{c}\text { GI: CLD } \\
\mathrm{N}=(\mathbf{9 5}) \\
\end{array}$} & \multirow[b]{2}{*}{$(\%)$} & \multicolumn{2}{|c|}{ GII: Non CLD } & \multicolumn{2}{|c|}{ Statistical analysis } \\
\hline & & & & $\mathrm{N}=(95)$ & $(\%)$ & $\mathrm{X}^{2}$ test & P-value \\
\hline \multirow{2}{*}{ Gender: } & Male & 63 & $(66)$ & 54 & $(57)$ & \multirow{2}{*}{1.8} & \multirow{2}{*}{0.179} \\
\hline & Female & 32 & (34) & 41 & (43) & & \\
\hline \multirow{3}{*}{ Age: } & $>15-35 y$ & 17 & $(18)$ & 31 & (33) & \multirow{3}{*}{5.49} & \multirow{3}{*}{0.064} \\
\hline & $>35-50 \mathrm{y}$ & 45 & $(47)$ & 36 & $(38)$ & & \\
\hline & $>50 \mathrm{y}$ & 33 & $(35)$ & 28 & $(29)$ & & \\
\hline \multirow{2}{*}{ Residence: } & Rural & 62 & (65) & 71 & (75) & \multirow{2}{*}{2.03} & \multirow{2}{*}{0.154} \\
\hline & Urban & 33 & (35) & 24 & $(25)$ & & \\
\hline
\end{tabular}


Table (2): Aetiological classification of CLD patients.

\begin{tabular}{|l|c|c|}
\hline \multicolumn{1}{|c|}{ Type of liver disease } & $\mathbf{N = ( 9 5 )}$ & $(\mathbf{\%})$ \\
\hline Liver cirrhosis & 43 & 45 \\
\hline Chronic hepatitis C & 24 & 25 \\
\hline Chronic hepatitis B & 13 & 14 \\
\hline Nonalcoholic fatty liver (NASH) & 10 & 11 \\
\hline Autoimmune hepatitis & 5 & 5 \\
\hline
\end{tabular}

Table (3): Clinical manifestations among CLD and Non-CLD studied cases.

\begin{tabular}{|l|c|c|c|c|c|c|c|c|}
\hline \multirow{2}{*}{$\begin{array}{l}\text { Clinical } \\
\text { Presentations }\end{array}$} & \multicolumn{2}{|c|}{ CLD } & \multicolumn{2}{c|}{ Non CLD } & \multicolumn{2}{c|}{ Total N=190 } & \multicolumn{2}{|c|}{ Statistical analysis } \\
\cline { 2 - 10 } & $\mathbf{N = ( 9 5 )}$ & $\mathbf{( \% )}$ & $\mathbf{N}=\mathbf{( 9 5 )}$ & $\mathbf{( \% )}$ & No. & $\mathbf{( \% )}$ & \multicolumn{2}{|c|}{} \\
\cline { 3 - 10 } & & & & & & & $\boldsymbol{X}^{\mathbf{2}}$ & P-value \\
\hline Diarrhea & 61 & $(64)$ & 37 & $(39)$ & 98 & $(52)$ & 12.14 & $0.000^{*}$ \\
\hline Abdominal pain & 31 & $(33)$ & 17 & $(18)$ & 48 & $(25)$ & 5.46 & $0.019^{*}$ \\
\hline Fever & 15 & $(16)$ & 6 & $(6)$ & 21 & $(11)$ & 4.3 & $0.037^{*}$ \\
\hline Nausea, vomiting & 11 & $(12)$ & 8 & $(8)$ & 19 & $(10)$ & 0.53 & 0.47 \\
\hline Bloating, flatulence & 35 & $(37)$ & 21 & $(22)$ & 56 & $(29)$ & 4.96 & $0.026^{*}$ \\
\hline
\end{tabular}

*Significant differences at $\mathrm{P}$-value $<0.05$.

Table (4): Clinical manifestations and sonographic findings in patients with chronic liver diseases.

\begin{tabular}{|l|c|c|}
\hline \multicolumn{1}{|c|}{$\begin{array}{c}\text { Clinical manifestations and sonographic } \\
\text { findings }\end{array}$} & N=(95) & (\%) \\
\hline Hepatomegaly & 30 & 32 \\
\hline Splenomegaly & 54 & 57 \\
\hline Lower limb oedema & 19 & 20 \\
\hline Ascites & 22 & 23 \\
\hline Jaundice & 24 & 25 \\
\hline Weight loss & 63 & 66 \\
\hline
\end{tabular}

Table (5): Types of diarrhea among diarrheic cases in the studied groups:

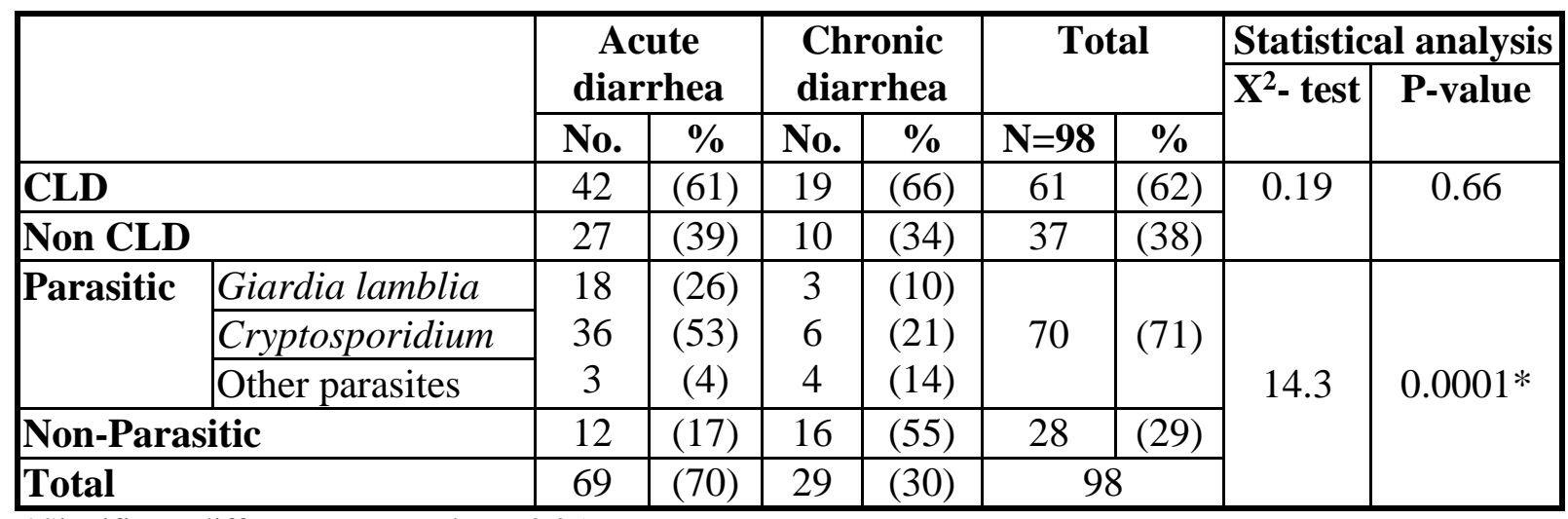

*Significant differences at $\mathrm{P}$-value $<0.05$. 
Table (6): Characters of diarrheic stool among diarrheic cases in the studied groups:

\begin{tabular}{|c|c|c|c|c|c|c|c|c|}
\hline & \multicolumn{2}{|c|}{ Acute diarrhea } & \multicolumn{2}{|c|}{$\begin{array}{l}\text { Chronic } \\
\text { diarrhea }\end{array}$} & \multicolumn{2}{|c|}{ Total $(\mathrm{N}=98)$} & \multicolumn{2}{|c|}{ Statistical analysis } \\
\hline & No. & $\%$ & No. & $\%$ & No. & $\%$ & $\mathrm{X}^{2}$ - test & P-value \\
\hline Mucoid & 3 & (4) & 5 & (17) & 8 & $(8.2)$ & \multirow{4}{*}{21.8} & \multirow{4}{*}{$0.000 *$} \\
\hline Soft (lacking fibers) & 8 & (12) & 4 & (14) & 12 & $(12.2)$ & & \\
\hline Watery & 51 & (74) & 8 & $(28)$ & 59 & $(60.2)$ & & \\
\hline Fatty (foamy) & 7 & (10) & 12 & $(41)$ & 19 & $(19.4)$ & & \\
\hline
\end{tabular}

*Significant differences at $\mathrm{P}$-value $<0.05$.

Table (7): Distribution of intestinal parasites among the studied groups:

\begin{tabular}{|c|c|c|c|c|c|c|c|c|}
\hline \multirow{3}{*}{ Parasites } & \multirow{2}{*}{\multicolumn{2}{|c|}{ CLD }} & \multirow{2}{*}{\multicolumn{2}{|c|}{ Non CLD }} & \multirow{2}{*}{\multicolumn{2}{|c|}{ Total }} & \multicolumn{2}{|c|}{ Statistical analysis } \\
\hline & & & & & & & \multirow[t]{2}{*}{$X^{2}$ test } & \multirow[t]{2}{*}{ P-value } \\
\hline & No. & $(\%)$ & No. & $(\%)$ & No. & $(\%)$ & & \\
\hline Giardia lamblia & 14 & (15) & 11 & (12) & 25 & (13) & \multirow{3}{*}{0.87} & \multirow{3}{*}{0.65} \\
\hline Cryptosporidium & 27 & (28) & 13 & (14) & 40 & (21) & & \\
\hline $\begin{array}{c}\text { Mixed Giardia and } \\
\text { Cryptosporidium }\end{array}$ & 5 & $(5)$ & 3 & (3) & 8 & (4) & & \\
\hline $\begin{array}{c}\text { Other parasites: } \\
\text { E.histolytica/dispar }\end{array}$ & 5 & (5) & 4 & (4) & 9 & (5) & \multirow{3}{*}{1.21} & \multirow{3}{*}{0.55} \\
\hline Blastocystis hominis & 3 & (3) & 1 & (1) & 4 & (2) & & \\
\hline H.nana & 1 & (1) & 2 & (2) & 3 & (2) & & \\
\hline Total & 55 & (58) & 34 & (36) & 89 & (47) & & \\
\hline
\end{tabular}

Table (8): Correlation between liver enzymes (ALT and AST) in giardial and cryptosporidial infected CLD cases.

\begin{tabular}{|l|c|c|c|c|}
\hline \multirow{2}{*}{ Liver enzymes } & \multicolumn{2}{|c|}{ ALT } & \multicolumn{2}{c|}{ AST } \\
\cline { 2 - 5 } Parasite & $\begin{array}{c}\text { Normal } \\
\text { No.(\%) }\end{array}$ & $\begin{array}{c}\text { Abnormal } \\
\text { No. }(\%)\end{array}$ & $\begin{array}{c}\text { Normal } \\
\text { No.(\%) }\end{array}$ & $\begin{array}{c}\text { Abnormal } \\
\text { No.(\%) }\end{array}$ \\
\hline Giardia lamblia: $\mathbf{N = 1 9}$ & $14(74)$ & $5(26)$ & $12(63)$ & $7(37)$ \\
\hline Cryptosporidium: $\mathbf{N = 3 2}$ & $13(41)$ & $19(59)$ & $11(34)$ & $21(66)$ \\
\hline $\mathbf{X}^{2}$ test & \multicolumn{2}{|c|}{5.23} & \multicolumn{2}{c|}{3.99} \\
\hline P-value & \multicolumn{2}{|c|}{$0.022^{*}$} & \multicolumn{2}{c|}{$046^{*}$} \\
\hline
\end{tabular}

*Significant differences at $\mathrm{P}$-value $<0.05$.

Table (9): Validity and Accuracy of ELISA in detection of Giardia lamblia and Cryptosporidium spp. among the examined cases:

\begin{tabular}{|c|c|c|c|c|c|c|}
\hline \multirow{3}{*}{$\begin{array}{l}\text { Microscopic } \\
\text { examination }\end{array}$} & \multicolumn{6}{|c|}{ ELISA } \\
\hline & \multicolumn{3}{|c|}{ Giardia lamblia } & \multicolumn{3}{|c|}{ Cryptosporidium spp. } \\
\hline & $+\mathrm{ve}$ & -ve & Total & +ve & -ve & Total \\
\hline +ve & 29 & 0 & 29 & 43 & 5 & 48 \\
\hline -ve & 4 & 157 & 161 & 0 & 142 & 142 \\
\hline Total & 33 & 157 & 190 & 43 & 147 & 190 \\
\hline Validity & \multicolumn{3}{|c|}{ 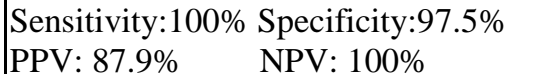 } & \multicolumn{3}{|c|}{\begin{tabular}{|ll} 
Sensitivity:89.6\% & Specificity: $100 \%$ \\
PPV: $100 \%$ & NPV: $96.6 \%$ \\
\end{tabular}} \\
\hline Accuracy & \multicolumn{3}{|c|}{$97.9 \%$} & & $98.4 \%$ & \\
\hline
\end{tabular}

PPV (positive predictive value), NPV (negative predictive value). 


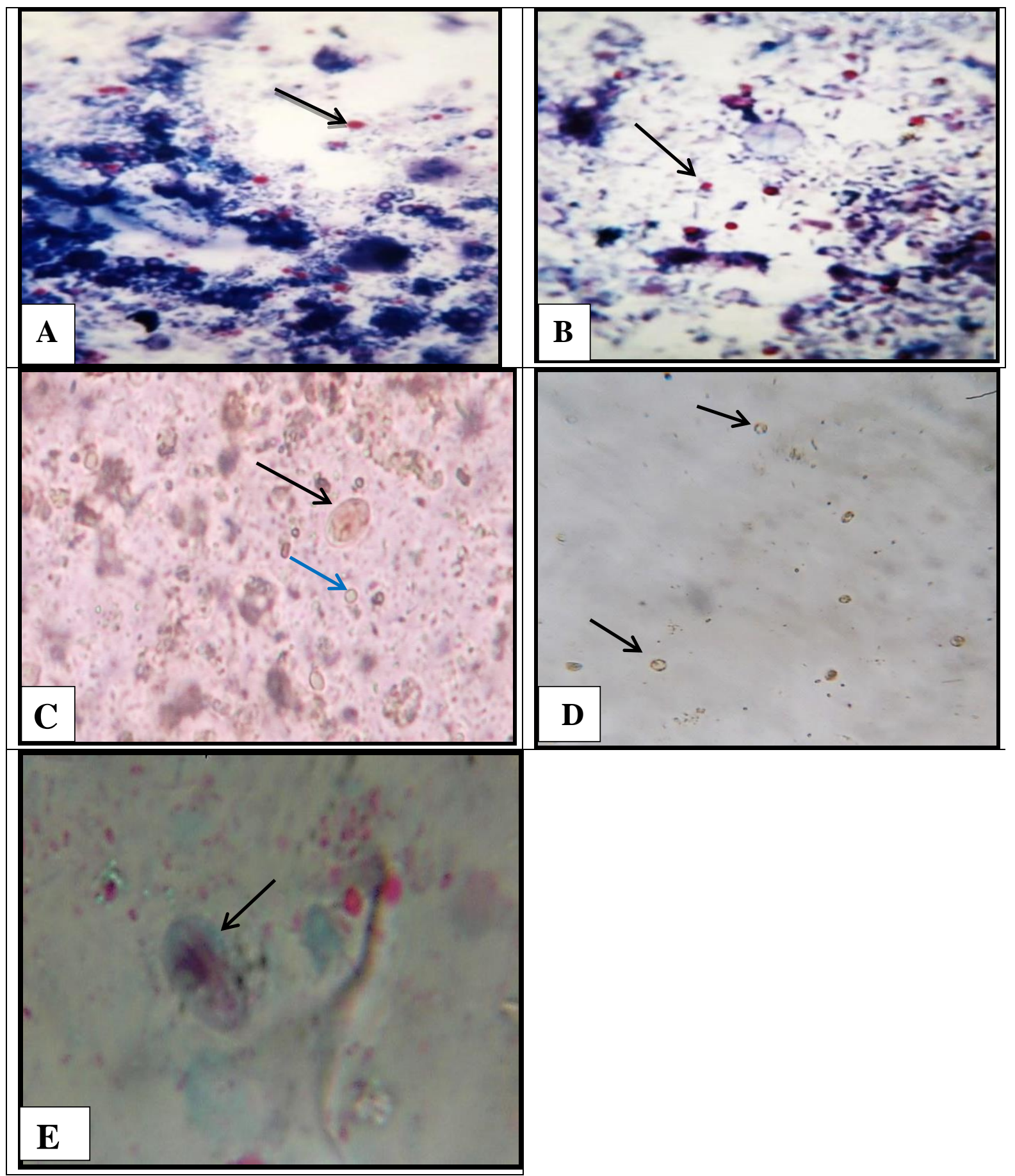

Figure (1): A-B) Cryptosporidium oocysts (arrows), Modified Ziehl-Neelsen stain (X1000). C) Giardia lamblia cyst (black arrow), and Cryptosporidium oocyst (blue arrow), Direct wet mount (X1000). D) Blastocystis spp., Direct wet mount smear (X1000). E) Giardia lamblia cyst (black arrow), Trichrome stain (X1000). 


\section{DISCUSSION}

In chronic Liver diseases (CLD), cirrhosis as a sequel and their major complications are chief roots of worldwide morbidity and mortality. In general, without efficient treatment, all types of chronic hepatitis will finally progress to cirrhosis, liver failure, or hepatocellular carcinoma (HCC) [24]. Cirrhosis due to any etiology disrupts the homeostatic role of liver. Cirrhosis-associated immune dysfunction leads to alterations in both innate and acquired immunity, which prone patient with cirrhosis to multiple infections or activation of any dormant infection [25].

An intimate pathophysiology of different infections was confined to liver tissue. Worsening of liver function may be triggered by Schistosoma-granulomatous inflammatory reaction around trapped Schistosoma eggs. Viral hepatitis, malaria, HIV infection, and mixed Schistosoma species could also enhance liver tissue damage with inflammatory/fibrotic responses, leading to induced liver injury and disease progression [26].

In the present study, after history taking, clinical, sonographic and laboratory confirmation, nearly half of CLD non admitted cases were represented by liver cirrhosis $(45 \%)$ followed by chronic hepatitis C [HCV] (25\%), and chronic hepatitis B $[\mathrm{HBV}]$ in $(14 \%)$, while non-alcoholic fatty liver (11\%) and autoimmune hepatitis (5\%). These results were not in accordance with that reported by Sabah and El-Lessi [27] who found that liver cirrhosis was represented in $36 \%$ of adult patients with CLD while non-alcoholic fatty liver was represented in $64 \%$ of those patients. Also, our results disagree with that obtained by Elshazly et al. [28] who reported that the etiologies of CLD in 50 children were biliary atresia in (24 $\%$ ), autoimmune hepatitis in (22\%), chronic $\mathrm{HCV}$ in $(10 \%)$. Also Larrosa-Haro et al. [29] and Delghani et al. [30] found that biliary atresia was the most evident etiology of CLD in pediatric patients (27.8 and $27.7 \%$, respectively). Moreover, Chaabouni et al. [31] documented biliary cirrhosis with different etiologies where biliary atresia was the most evident cause $(40 \%)$ then $(17 \%)$ for each of post- hepatitic and metabolic cirrhosis. These differences in the disease distribution among the previous mentioned studies may be attributed to the different etiologies of CLD among the different ages (children, adolescents and adults) [32].
Gastrointestinal symptoms are common in CLD and their pathophysiology may be related to many factors as the severity of liver disease, psychological distress, and GIT dysfunction. Delayed small bowel transit in patients with liver cirrhosis may lead to overgrowth of microorganisms, which could contribute to the symptoms of abdominal pain and diarrhea [33].

In our study, all GIT manifestations were significantly different between CLD and nonCLD patients except for abdominal pain. This agreed with Fritz and Hammer [34] who found a significant difference between the cirrhotic patients and the control group regarding the GIT manifestations. The most common GIT symptoms reported were abdominal bloating, abdominal pain and diarrhea.

Human intestinal parasitic infections are series of public health problems in developing countries [35]. Even in communities where adequate sanitary conditions and higher educational levels are present, some of these intestinal parasites play an important role in causing diseases especially in specific groups such as immunocompromised patients [10]. This study found an intimate relationship between the occurrence of chronic hepatic insult and the presence of intestinal parasitic infection in such patients. We found that CLD patients had higher rates of enteric parasitic infections than non CLD patients this could be explained by the major role of the liver in protection against infection. However, the most evident parasitic infections were Cryptosporidium spp. and Giardia lamblia protozoa. This may be attributed to the extension of cryptosporidiosis pathology to the biliary tract and liver especially in immunocompromised [13], and the duodenal habitat of giardiasis, though superficial, but it may aggravate biliary tract and even hepatic affection by causing dehydration and fatty diarrhea.

In this study, the overall parasitic infection rate detected in both studied groups was $(47 \%)$. In the CLD group (58\%) of cases were infected as follow: Cryptosporidium (28\%), Giardia (15\%), mixed Cryptosporidium and Giardia (5\%), Entamoeba histolytica spp. (5\%), Blastocystis hominis (3\%) and H. nana (1\%). In the non-CLD group $36 \%$ of cases were infected as follow: Cryptosporidium (14\%), Giardia (12\%), mixed Cryptosporidium and Giardia (3\%), E. histolytica spp. (4\%), Blastocystis hominis (1\%) and H.nana (2\%). These results disagreed with 
Sabah and El-Lessi [27] who studied the prevalence of intestinal parasites among adult CLD patients and found that E. histolytic was the commonest parasite (48\%) compared to; $G$. lamblia (4\%), A. lumbricoides (5.3\%), E. vermicularis (2.7\%), and S. mansoni (1.3\%). Also, our results were not in concordance with that reported by El-Shazly et al. [28] who reported Entamoeba histolytica spp.(16\%), Giardia lamblia (14\%), Blastocystis hominis (14 $\%)$, Cryptosporidium parvum (10 \%), E. histolytica and G. lamblia (2\%), E. histolytica and $B$. hominis (2\%), G. lamblia and B. hominis (2\%), B. hominis and Entamoeba coli (2\%), Microsporidia (2\%) in CLD patients. Hegab et al. [11] on their study on 80 CLD patients, found that G. lamblia was the most common detected organism which present in (45\%) of cases followed by $E$. histolytica spp. (37.5\%), B. hominis (25\%) and Microsporidia (22.5\%).

Cryptosporidium parvum is one of the most common opportunistic intestinal coccidia in vertebrates. In developing countries, it is highly documented as an imperative source of diarrheal illness in malnourished, debilitated cases with chronic diseases with strong association among viral hepatitic patients. Cryptosporidiosis is mainly characterized by mucoid, watery diarrhea of six to eight episodes per day. This Diarrhea is usually self-limiting in immunocompetents, but with lethal outcomes in immunocompromised. Being affecting the overall health of such cases, chief advances have been conveyed in screening, and early detection with precise diagnostic tools $[12,36]$. In this study, the main parasite detected was Cryptosporidium spp. in (28\%) of CLD patients, while it comprises (14\%) only in nonCLD patients. This was nearly similar to results obtained by Mousa et al. [37] who detected Cryptosporidium infection in $30 \%$ of CLD patients versus $14 \%$ of controls. Also, Shrestha et al. [38] found Cryptosporidium infection in $20 \%$ of CLD cases.

Giardia lamblia is an intestinal flagellated protozoan parasite causing giardiasis that has a global distribution and is common in both children and adults. Clinically, the infection varies from being asymptomatic to acute offensive fatty diarrhea, nausea, epigastric pain, and weight loss [39]. In our study, Giardia lamblia infection was reported in (15\%) of CLD patients and (12\%) of non-CLD patients. These results were comparable with Sabah and El-Lessi [27], El-Shazly et al. [28] and Hegab et al. [11] who reported Giardia infection in 4\%, 14\%, and $45 \%$ of CLD respectively.

Most cases of Giardia lamblia infection in CLD group had normal levels of ALT and AST (74\%, $63 \%$ ) respectively while most cases of Cryptosporidium spp. infection had elevated ALT and AST $(59 \%, 66 \%)$ respectively with significant difference between the two infected groups. These results disagreed with Mousa et al. [37] who did not find any significant difference in serum liver enzymes between CLD patients with and without Cryptosporidium infection.

For Giardia and Cryptosporidia spp., the most widely used antigen detection immunoassays were the direct fluorescent-antibody (DFA) tests [40], which could detect intact organisms, and enzyme immunoassays (EIAs), detecting soluble stool antigens $[\mathbf{4 1 , 4 2 ]}$. In the current study, assessment of cryptosporidiosis and giardiasis was done parasitologically by Modified ZiehlNeelsen (MZN) staining and Trichrome staining of stool samples respectively (the reference standard tests) $[\mathbf{2 0 , 1 9 ]}$ and both infections were confirmed by RIDASCREEN ELISA [21]. ELISA detected more cases of Giardia infection than microscopy (4 cases not detected by microscopy). This may be attributed to the intermittent excretion of Giardia protozoan in stool [43], while in Cryptosporidium infection ELISA failed to detect five cases which were detected by microscopy. This could be explained by the capability of RIDASCREEN ELISA to detect Cryptosporidium parvum spp. only while, human cryptosporidiosis is generally caused by Cryptosporidium hominis spp., beside many other different genotypes of Cryptosporidium [44]. The sensitivity, specificity, PPV, NPV and diagnostic accuracy of ELISA regarding Giardia lamblia infection were as follow $(100 \%, 97.5 \%$, $87.9 \%, 100 \%$, and $97.9 \%$ ) respectively. These results were near to that reported by Singhal et al. [45] who detected RIDASCREEN Giardia ELISA sensitivity $96.8 \%$, Specificity $91.6 \%$, PPV 70.6\%, NPV $99.3 \%$ and diagnostic accuracy $92.5 \%$. Also, our results were comparable to studies performed by DuqueBeltran et al. [46], Ozekinci et al. [47] and Guimarães and Sogayar [48], where the sensitivity of ELISA for Giardia was $100 \%$, $96.4 \%$ and $82 \%$, respectively, and the specificity was $95 \%, 80.8 \%$ and $39 \%$, respectively.

The sensitivity, specificity, PPV, NPV and diagnostic accuracy of ELISA regarding 
Cryptosporidium spp. infections were as follow (89.6\%, 100\%, 100\%, 96.6\%, and 98.4\%) respectively. These results were near to that reported by El-Tantawy et al. [49] who detected RIDASCREEN Cryptosporidium ELISA sensitivity $78 \%$, specificity $100 \%$, PPV $100 \%$, NPV $98 \%$ and diagnostic accuracy $98 \%$. Similarly, Weitzel et al. [21] reported ELISA sensitivity $82.4 \%$, Specificity $100 \%$, PPV $100 \%$ and NPV $98.5 \%$

\section{CONCLUSION}

The high detection rates of intestinal parasitic infections among CLD outpatients who were mostly complaining of GIT manifestations e.g. diarrhea should direct our attention towards elevating their general health status with better utilization of purified water supplies and good management for hepatic patients. Rural areas of Zagazig province were found to be more vulnerable to acquire such infections, mainly cryptosporidiosis and giardiasis; confirmed by immunological techniques that provide a superior diagnostic tool than conventional direct microscopic examination of fecal samples for their better follow-up and management.

Ethical approval: This study was reviewed and approved by the Institutional Review Board of the faculty of Medicine, Zagazig University, Egypt.

\section{Conflicts of interest: None.}

Funding: None

\section{REFERENCES}

1. Mann J. Epigenetics in Liver Disease: Involvement of Oxidative Stress. Chapter 15; Quoted from Section II Liver Pathophysiology 2017; (15): 199-211.

2. Kurokawa T, Ohkohchi N. Role of Platelet, Blood Stem Cell, and Thrombopoietin in Liver Regeneration, Liver Cirrhosis, and Liver Diseases. Quoted from Stem Cells and Cancer in Hepatology, From the Essentials to Application 2018; 9: 159-177.

3. Abenavoli L, Corpechot C, Poupon R. Elastography in hepatology. Can J Gastroentrol 2007; 21(12):839-842.

4. Plauth M, Bernal W, Dasarathy S, Merli M, Plank LD, Schütz T, Bischoff SC. Guideline on clinical nutrition in liver disease. European Society for Clinical Nutrition and Metabolism. ESPEN Clinical Nutrition; 2019, 1-37.
5. Polaris Observatory HCV Collaborators. Global prevalence and genotype distribution of hepatitis C virus infection in 2015: a modelling study. Lancet Gastroenterol Hepatol. 2017 Mar;2(3):161-176.

6. Gomaa A, Allam N, Elsharkway A, El Kassas M, Waked I. Hepatitis C infection in Egypt: prevalence, impact and management strategies. Review, Hepatic Medicine: Evidence and Research HMER; 2017, 9-017.

7. Wiest R, Garcia-Tsao G. Bacterial translocation (BT) in cirrhosis. Hepatology 2005; 41:422-433.

8. Atia MM, Abdul Fattah MM, Abdel Rahman HA, Mohammed FA, Al-Ghandour AMF. Assessing the Efficacy of Nitazoxanide in Treatment of Cryptosporidiosis Using PCR Examination. Journal of the Egyptian Society of Parasitology 2016; 46(3):683-692.

9. Rossignol JF. Cryptosporidium and Giardia: Treatment options and prospects for new drugs. Experimental Parasitology 2010; 124: 45-53.

10. Drawany ZEE, Saleh SHA, Etewa SE, Ibrahim SM. Prevalence of intestinal parasites among type 1 diabetic patients in pediatrics Zagazig university hospital. Endocrinol Metab Int J 2019; 7(6):171-179.

11. Hegab MA, Zamzam SM, Khater NM, Tawfeek DM, Abdel-Rahman HM. Opportunistic intestinal parasites among children with chronic liver disease. J Egypt Soc Parasitol 2003; 33(3):969977.

12. Wang B, Castellanos-Gonzalez A, White Jr AC. "Review: Novel drug targets for treatment of cryptosporidiosis". Journal of Expert Opinion on Therapeutic Targets 2020; 24(9): 915-922.

13. Al-Ghandour AMF, Yousef AM, Mohamed RMSM, Tealeb AM, Ahmed HK, Atwa HA, Farag TI. Prophylactic Anticryptosporidial Activity Of Atorvastatin Versus Nitazoxanide On Experimentally Infected Immunosuppressed Murine Models. Journal of the Egyptian Society of Parasitology 2020; 50(3) 535- 546.

14. Ali SA, Hill DR. Giardia intestinalis. Curr. Opin. Infect. Dis 2003, 16: 453-460.

15. World Health Organization "Diarrhoeal disease Factsheet"; 2017.

16. Barr W, Smith A. Acute Diarrhea in Adults. Am Fam Physician 2014; 89(3):180-189.

17. Juckett G, Trivedi R. Evaluation of Chronic Diarrhea. Am Fam Physician 2011; 84(10):11191126. 
18. Chessbrough M. Medical laboratory Manual for Tropical countries, 2nd ed., English Language Book society/ Tropical Health Technology/ButterWorths 1987; 548-577.

19. Garcia LS. Diagnostic medical parasitology, 4th edn. 2001, ASM Press, Washington, D.C.

20. Casemore DP, Armstrong M, Sands RL. Laboratory diagnosis of cryptosporidiosis. J. Clin. Pathol. 1985; 38:1337-1341.

21. Weitzel T, Dittrich S, Mo“hl I, Adusu E, Jelinek T. Evaluation of seven commercial antigen detection tests for Giardia and Cryptosporidium in stool samples. Clinical Microbiology and Infection 2006; 12 (7): 656-659.

22. Vagvala SH, O'Connor SD. Imaging of abnormal liver function tests. Clin Liver Dis (Hoboken); 2018; 11(5):128-134.

23. Peat J, Barton B. Medical statistics. A Guide to data analysis and critical appraisal. First Edition. Wiley-Blackwell; 2005, 113-119.

24. Wang FS, Fan JG, Zhang Z, Gao B, Wang HY. The global burden of liver disease: the major impact of China. Hepatology 2014; 60(6): 20992108.

25. Noor MT, Manoria P. Immune Dysfunction in Cirrhosis. J Clin. Transl. Hepatol. 2017; 5(1): 5058.

26. Cavalcanti MG, de Araujo-Neto JM, Peralta JM. Schistosomiasis: Clinical Management of Liver Disease. An Official Learning Resource of AASLD. Clinical Liver Disease 2015; 6, (3):5962.

27. Sabah AA, El-Lessi FME. Prevalence of common intestinal parasites in adult patients with chronic liver diseases associated with Helicobacter pylori infection. J. Egypt. Soc. Parasitol. (JESP) 2017; 47(3): 695-699.

28. El-Shazly LB, El-Faramawy AA, El-Sayed NM, Ismail KA, Fouad SM. Intestinal parasitic infection among Egyptian children with chronic liver diseases. Indian Society for Parasitology; 2013, J. Parasit. Dis. 2015; 39(1): 7-12.

29. Larrosa-Haro A, Hurtado-López E, Va'squezGaribay E. Liver function test predicts anthropometrical nutritional status in chronic liver disease. J Pediat Gastroenterol. Nutr. 2006; 43(4): 35-38.

30. Delghani SM, Gholami S, Bahador A, et al. Morbidity and mortality of children with chronic liver diseases who were listed for liver transplantation in Iran. Pediatr. Transplant 2007; 11(1): 21-23.
31. Chaabouni M, Bahloul S, Ben Romdhane W, Ben Saleh M, Ben Halima N, Chouchene C, et al. Aspects épidémiologiques, étiologiques et évolutifs des cirrhoses de l'enfant dans un pays en développement: expérience du service de pédiatrie du CHU de Sfax, Tunisie [Epidemiological, etiological and evolutionary aspects of children cirrhosis in a developing country: experience of the pediatric department of SFAX University hospital, Tunisia]. Tunis Med. 2007 Sep;85(9):738-43.

32. Pinto RB, Schneider ACR, Silveira TRD. Cirrhosis in children and adolescents: An overview. World J Hepatol. 2015; 7(3): 392-405.

33. Kalaitzakis E. Gastrointestinal dysfunction in liver cirrhosis. World J Gastroenterol. 2014; 20(40): 14686-14695.

34. Fritz E, Hammer J. Gastrointestinal symptoms in patients with liver cirrhosis are linked to impaired quality of life and psychological distress. Eur $J$ Gastroenterol Hepatol. 2009; 21:460-465.

35. Tegen D, Damtie D, Hailegebriel T: Prevalence and Associated Risk Factors of Human Intestinal Protozoan Parasitic Infections in Ethiopia: A Systematic Review and Meta-Analysis. $J$ Parasitol Res. 2020; 8884064.

36. Yu Z, Li F, Zeng Z, Huang Z, Fan Z, Jin Y, et al.: Prevalence and clinical significance of Cryptosporidium infection in patients with hepatitis B virus-associated acute-on-chronic liver failure. Int J Infect Dis. 2011, 15 (12): 845-848.

37. Mousa N, Abdel-Razik A, El-Nahas H, El-Shazly A, Abdelaziz M, Nabih M, Hamed M, Eissa M, Effat N, Eldars W. Cryptosporidiosis in patients with diarrhea and chronic liver diseases. J. Infect. Dev. Ctries. 2014, 15; 8(12):1584-90.

38. Shrestha S, Larsson S, Serchand J, Shrestha S. Bacterial and cryptosporidial infection as the cause of chronic diarrhea in patients with liver disease in Nepal. Trop. Gastroenterol. 1993; 14: 55-58.

39. Feng Y, Xiao L: Zoonotic potential and molecular epidemiology of Giardia species and giardiasis. Clin. Microbiol. Rev. 2011; 24: 110-40.

40. Garcia LS, Shum AC, Bruckner DA: Evaluation of a new monoclonal antibody combination reagent for direct fluorescence detection of Giardia cysts and Cryptosporidium oocysts in human fecal specimens. J. Clin. Microbiol. 1992; 30:3255-3257. 
41. Garcia LS, Shimizu RY: Detection of Giardia lamblia and Cryptosporidium parvum antigens in human fecal specimens using the ColorPAC combination rapid solid-phase qualitative immunochromatographic assay. J. Clin. Microbiol. 2000; 38:1267-1268

42. Garcia LS, Shimizu RY: Evaluation of nine immunoassay kits (enzyme immunoassay and direct fluorescence) for detection of Giardia lamblia and Cryptosporidium parvum in human fecal specimens. J. Clin. Microbiol. 1997; $35: 1526-1529$.

43. Chakarova B. Comparative evaluation of the diagnostic methods for detection of Giardia intestinalis in human fecal samples. Trakia J Sci. 2010; 8:174-179.

44. Molloy SF, Smith HV, Kirwan P, Nichols RA, Asaolu SO, Connelly L, Holland CV. Identification of a high diversity of Cryptosporidium species genotypes and subtypes in a pediatric population in Nigeria. Am J Trop Med Hyg. 2010; 82(4):608-613.
45. Singhal S, Mittal V, Khare V, Singh YI. Comparative analysis of enzyme-linked immunosorbent assay and direct microscopy for the diagnosis of Giardia intestinalis in fecal samples. Indian J Pathol Microbiol. 2015; 58:6971.

46. Duque-Beltrán S, Nicholls-Orejuela RS, ArévaloJamaica A, Guerrero-Lozano R, Montenegro S, James MA. Detection of Giardia duodenalis antigen in human fecal eluates by enzyme-linked immunosorbent assay using polyclonal antibodies. Mem Inst Oswaldo Cruz. 2002; 97:1165-1168.

47. Ozekinci T, Uzun A, Suay A, Elçi S, Akpolat N, Atmaca S. Comparison of microscopy and EIA in the diagnosis of Giardia intestinalis in stool specimens. Turkiye Parazitol Derg 2005; 29:8992.

48. Guimarães S, Sogayar MI. Detection of antiGiardia lamblia serum antibody among children of day care centers. Rev Saude Publica 2002; 36:63-68.

49. El-Tantawy NL, El-Nahas HA, Sultan DM. Diagnosis of Giardia and Cryptosporidium Infection in Children with Diarrheal Illness Using Rapid Immunochromatographic Assay. Archives of Clinical Microbiology 2012; 3(1):1. 